\title{
Cloud/Fog Computing System Architecture and Key Technologies for South-North Water Transfer Project Safety
}

\author{
Yaoling Fan $\mathbb{D}^{1},{ }^{1}$ Qiliang Zhu, ${ }^{2}$ and Yang Liu ${ }^{2}{ }^{2}$ \\ ${ }^{1}$ School of Water Conservancy \& Environment, Zhengzhou University, Zhengzhou, China \\ ${ }^{2}$ School of Information Engineering, North China University of Water Resources and Electric Power, Zhengzhou, China \\ Correspondence should be addressed to Yaoling Fan; fyl@ncwu.edu.cn
}

Received 4 June 2017; Accepted 17 September 2017; Published 16 January 2018

Academic Editor: Shangguang Wang

Copyright (c) 2018 Yaoling Fan et al. This is an open access article distributed under the Creative Commons Attribution License, which permits unrestricted use, distribution, and reproduction in any medium, provided the original work is properly cited.

\begin{abstract}
In view of the real-time and distributed features of Internet of Things (IoT) safety system in water conservancy engineering, this study proposed a new safety system architecture for water conservancy engineering based on cloud/fog computing and put forward a method of data reliability detection for the false alarm caused by false abnormal data from the bottom sensors. Designed for the South-North Water Transfer Project (SNWTP), the architecture integrated project safety, water quality safety, and human safety. Using IoT devices, fog computing layer was constructed between cloud server and safety detection devices in water conservancy projects. Technologies such as real-time sensing, intelligent processing, and information interconnection were developed. Therefore, accurate forecasting, accurate positioning, and efficient management were implemented as required by safety prevention of the SNWTP, and safety protection of water conservancy projects was effectively improved, and intelligential water conservancy engineering was developed.
\end{abstract}

\section{Background}

Water conservancy engineering presents the national fundamental industry and is vital in national economic development. The construction of water conservancy projects is generally large in scale, with high investment, wide geographical distribution, and decentralized management, in a long construction period and contains a large amount of information. It may be also affected by unfavorable terrain conditions, complex geological structure, and flood, which increase the difficulty in supervision. Therefore, the construction of Internet safety information system is of great significance for improving safety monitoring and ensuring project safety and water quality safety in water conservancy engineering.

With the rapid development of Internet of Things (IoT) technology in recent years, an important source of big data is the tremendous amount of data that are collected, transferred, stored, and processed through the IoT system [1-3]. Due to the general dense geographical distribution of water conservancy projects, the IoT-based data in water conservancy engineering have typical geographical attributes.
Taking the Middle Route of the SNWTP as an example, its main line starts at Taocha Sluice of Danjiangkou Reservoir, crosses the Yangtze River, Huaihe River, Yellow River, and Haihe River, passes through Provinces of Henan, Hebei, Beijing and Tianjin, and provides water for tens of large- and medium-sized cities, like Pingdingshan, Xuchang, Zhengzhou, Jiaozuo, Xinxiang, Hebi, Anyang, Handan, Xingtai, Shijiazhuang, Baoding, Beijing, Tianjin, and so on. Therefore, it is necessary to build the safety information network system for the water conservancy project.

Combined with the research work of the National Major Project "Research and Development of Sensor Network Technology Oriented to the Safety of the South-North Water Transfer Project (SNWTP)," this study investigated the cloud/fog network system architecture for project safety of the Middle Route of SNWTP. This paper first analyzed the application and problems of cloud computing in the water conservancy industry and then proposed the cloud/fog network architecture for project safety of the Middle Route of SNWTP. It is also responsible for the occurrence of false anomaly data from the bottom sensors of the water 
conservancy project network; a method of data reliability detection for the false alarm is put forward.

\section{Application of Cloud Computing in Water Conservancy Industry}

Cloud computing, as a new generation of computing architecture with strong scalability, has become a basic platform to support big data applications, and it effectively satisfies the growing data processing and storage requirements in areas such as water conservancy engineering [4-6]. In terms of service pattern, Sun et al. designed and implemented the hydrological simulation and water resources management in the cloud computing model for the specific application requirements of distributed hydrological modeling and water resources monitoring [7, 8]. Ari and Muhtaroglu designed the cloud service framework of finite element simulation and implemented some modules [9]. Zhang and Wang proposed the technology architecture of an integrated management information platform in water conservancy engineering based on cloud computing technology [10]. R. J. Yang and Y. $\mathrm{J}$. Yang set up an experimental platform for water resources management system based on private cloud service [11]. Zhou et al. studied the cloud computing platform system and its application in water resources research [12].

The aforementioned studies provide technical support and implementation ideas for solving some water conservancy problems, especially in terms of efficient use of resources, scalability, and cost savings. However, the studies also demonstrated the limitations of the cloud computing architecture. In the application of big data of IoT in water conservancy engineering, especially due to high requirement for latency and dense geographical distribution of the water resources data, the problems are presented as follows.

(1) It is unable to meet the requirement of low latency in early warning applications such as water quality safety and human safety.

Traditional cloud computing causes large round-trip delays because the enormous amounts of data generated from the distributed sources have to be transferred to the computing center for processing. The situation will be more severe when the amount of data and transferring speed exceed the current capacity of the equipment, so it may take several days or weeks to transfer terabytes of data to the cloud and bring huge administrative costs.

(2) The enormous amounts of water conservancy engineering data are geographically distributed.

Water conservancy projects generally cover a large area. Taking the Middle Route of the SNWTP as an example, there is a management station in each bypassed city, and each management station has a number of sluice gate stations. The sensors of water conservancy facilities are widely distributed, so that the centralized cloud computing method is not suitable.

(3) The network of IoT is generally complex, and it does always meet the requirements of transmission bandwidth and reliability in cloud computing.

The computing power of the facilities at sluice stations is limited, largely restricted by the energy supply in the field and poor onsite installation environment, so the bandwidth and reliability of the formed network architecture are far from satisfying the requirements of traditional cloud computing.

\section{Characteristics of Fog Computing Architecture}

In order to take advantage of the flexibility of cloud computing architecture as well as to solve the above problems encountered in the IoT big data applications, Cisco Inc. proposed the concept of fog computing in 2012.

Fog computing is conceptually the extension of cloud computing. Fog computing was named after cloud computing due to the concept that "cloud at ground level is known as fog." Compared with cloud computing, the architecture used for fog calculation is more distributed and closer to network edges. Fog computing arranges data, data processing, and applications at network edges, which is unlike cloud computing that keeps them almost entirely in the cloud. The storage and processing of data are more reliable on local facilities than servers in fog computing. Therefore, cloud computing is a new generation of centralized computing, while fog computing is a new generation of distributed computing, which is in line with the "decentralization" feature of Internet $[13,14]$.

Fog computing is mainly based on small cloud such as personal, private, and enterprise cloud, while cloud computing is mainly based on IT services, public cloud. Fog calculation is powerful in large quantity and emphasizes the quantity, where the single computing node plays an important role, while cloud computing emphasizes the overall computing power, which is typically calculated by a bunch of concentrated high-performance computing facilities. Fog calculation expands the network-computing model of cloud computing and extends the network computing from the center to the edges of the network, as shown in Figure 1, which is therefore more widely used in a variety of applications [15-18].

The noticeable characteristics of fog calculation are as follows.

(1) Low latency and position sensing characteristics as fog computing locates at edges: it is of significance to the current informatics IoT in water conservancy engineering; for example, low latency is required in sudden floods, water pollution, and personal safety.

(2) Dense geographical distribution: this coincides with the wide distribution of water management of the SNWTP.

(3) Enormous amounts of nodes: a large-scale sensor network with numerous network nodes can be used to monitor the environment.

(4) Adaptability to access to mobile devices: mobile devices in fog computing can communicate directly with each other without transferring to the cloud or station, so that fog computing is highly adaptable to mobile devices, for example, mobile water quality monitoring and water level monitoring [19].

(5) High real-time feature: fog computing supports computation and processing of data at network edges with a low latency [20]. 


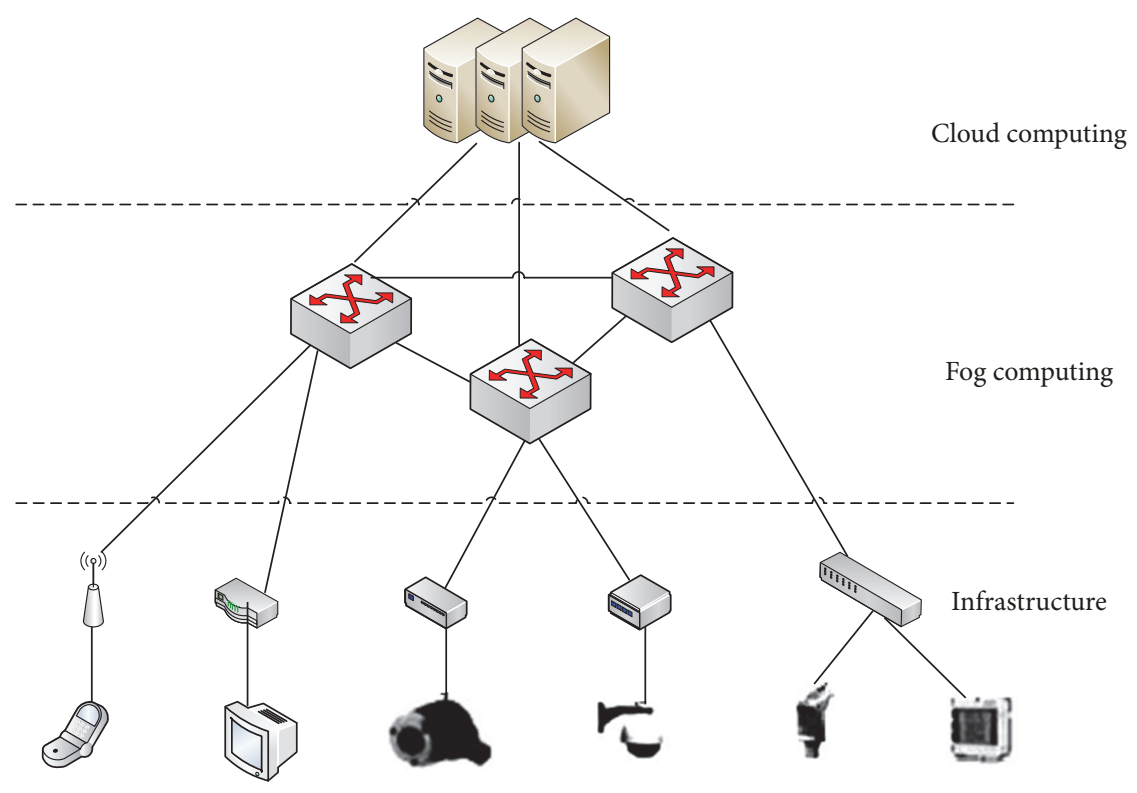

FIGURE 1: Fog between edge and cloud.

(6) Supportive to diverse heterogeneous software and hardware: fog computing devices are inherently heterogeneous and are deployed in different locations in the environment, such as cores, edges, access networks, and endpoints.

\section{Safety System Architecture for the SNWTP}

4.1. Monitoring Object of the SNWTP. The SNWTP is a strategic project in China. It is the infrastructure to optimize water resources allocation and promote regional coordinated development. Therefore, it is extremely important to ensure project safety, water supply safety, and human safety. Taking the Middle Route of the SNWTP as an example, the specific attributes of the three types of safety monitoring are as follows.

4.1.1. Project Safety Monitoring. The main line of the Middle Route is $1432 \mathrm{~km}$ in length, including 318 control buildings, 1256 bridges, and 469 left bank drainage structures. Therefore, it is necessary to prevent and control safety problems due to channel seepage or leakage, seepage in inverted siphons or dark culverts, and uneven settlement or deformation of buildings. The monitoring task is challenging.

4.1.2. Water Supply Safety Monitoring. For the Middle Route of the SNWTP, it requires a Class II water quality when the water is transferred to Beijing. However, it is difficult to ensure the water quality at the source region and to control the point and nonpoint pollution. In addition, sudden accidents easily cause water pollution. The quantity safety level is also high. Therefore, high accuracy is required for realtime gates operation, overall water surface profile control, and water level and flow monitoring. A wide range of meteorological and hydrological monitoring is also needed.
4.1.3. Human Safety Monitoring. Because the Middle Route of the SNWTP extensively crosses over local highways, railways, and rivers, and open management strategies are adapted; therefore, the risk of external invasion is high. When outsiders enter the channel to play with water or go fishing, hidden dangers exist, for example, dawning of people or stealing of important facilities, which may result in water conveyance accidents and threat human safety. Rigorous monitoring of human safety is necessary.

4.2. A Cloud/Fog Network System Architecture. There are more than 5,000 wired or wireless sensors in the Middle Route of SNWTP, with high-definition camera devices installed at every $500 \mathrm{~m}$. These basic data acquisition devices and related computing resources and network resources represent the IoT nodes of the Middle Route of SNWTP. Based on the enormous amount of data generated by IoT, relevant big data analysis is conducted to apply in the safety prevention of water conservancy projects and to solve problems that are not able to or difficult to be solved by traditional methods. This study presents a cloud/fog network system architecture, as shown in Figure 2, oriented to the safety of the Middle Route of the SNWTP in water conservancy engineering.

4.2.1. Infrastructure Layer. This layer is the information input point of the water conservancy project safety platform, including various types of sensors and some network physical devices for water conservancy data acquisition. The sensors are deployed in the scattered geographical areas according to the application needs. Real-time monitoring of the corresponding water conservancy projects is implemented by sensor network, IoT technology, remote sensing technology, video capture technology, and so on. The sensors are mainly responsible for detection and collection of basic data such as osmotic pressure, water level, water flow, and precipitation. 


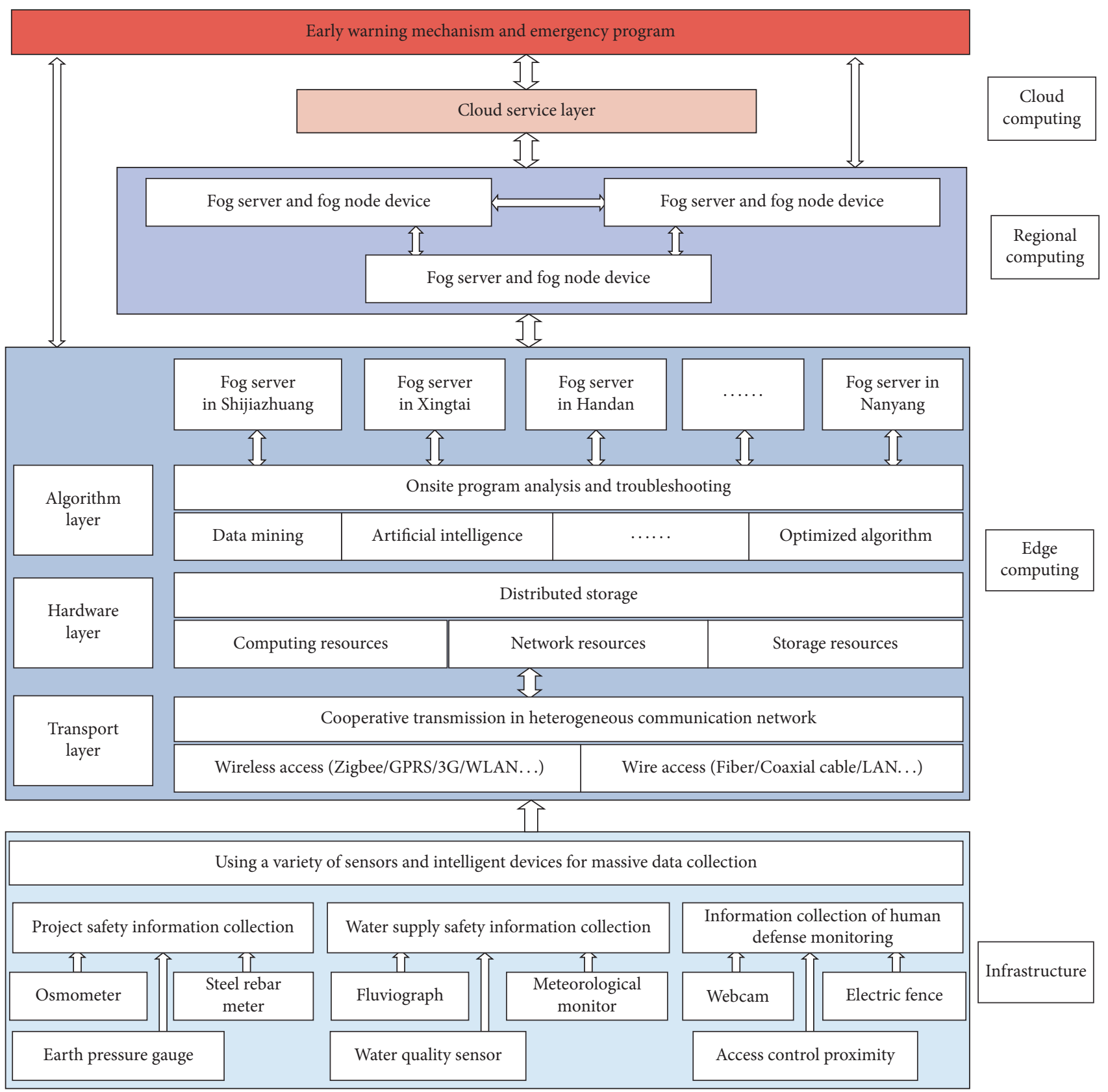

FIGURE 2: The cloud/fog network system architecture for project safety of the Middle Route of SNWTP.

The data are transferred to the jurisdictional water management center based on the IoT agreement in the form of data flow.

4.2.2. Edge Computing Layer. This layer is used for data storage, processing, and analysis at each water conservancy management center, which belongs to the computing edge unit in the whole network. The layer is divided into hardware layer and virtualization layer, including the corresponding hardware and software resources like network fog server, operating system, storage, database management, and so on. Among them, the fog server collects the data from the data collecting devices at water conservancy project sites, followed by cleaning, filtering, and fusion of the collected data.

The local computing mainly uses different data processing software and a variety of data analysis algorithms to process and analyze the real-time monitoring data stored at local hardware, in order to implement onsite low-latency real-time monitoring. Due to the large amount of safety monitoring data in the Middle Route of the SNWTP, the edge computing equipment can balance the load by distributed calculation of data from each region. Results used as a reference for management center can be obtained in a relatively short time; therefore, quick decision can be made and the data processing and transmission waiting time is largely reduced. 
4.2.3. Regional Management Layer. This layer can manage and compute the local data at each regional management center, acquire and share classified data of each region at the edge computing units, and implement the middle level computation. For example, the fog server at this layer can track, perform analysis, and forecast based on the abnormal water quality data of each region. The computational load and the time delay to the management center are relatively larger than those at the edges.

4.2.4. Cloud Computing Layer. Cloud computing allows a large number of computing tasks to share high-speed hardware resources through the establishment of large-scale computing center and virtualization technology, which can effectively reduce computing and hardware maintenance costs. The layer focuses more on the application of high latency data with a large number of data types and complex computational model. It is used for safety risk assessment for the entire SNWTP and provides a computation platform for large regional intelligential water conservancy projects.

4.2.5. Early Warning Mechanism and Emergency Program. The early warnings of different level respond to different contingency plans. Optimized early warning program for safety of the Middle Route of SNWTP is developed, and early warning for client is constructed. The Android or Apple phone and computer are used as the early warning release end.

The cloud/fog network system architecture for project safety of the Middle Route of SNWTP adapts to the characteristics of wide geographical distribution and long-distance water supply monitoring in the project, which satisfies the real-time local computing of each water conservancy management center and regional management requirements, and provides a platform of intelligent management and safety risk assessment for the entire SNWTP. This architecture is adapted to multilevel requirements, achieves a unified deployment, and better meets the needs of security monitoring of the Middle Route of SNWTP.

\section{Key Technologies}

The data from the bottom sensors has the characteristics of large amount and various types in the edge computing and region computing. Once the data from some sensors is pseudo-abnormal, it is bound to cause false alarm. In order to find out the real abnormal data from a large number of data and reduce false alarm rate, the data should be authenticated and analyzed.

The monitoring data of the Middle Route of the SNWTP is mainly from wired or wireless multitype sensors. The security detection mainly analyzes the security of the detected object based on alarm threshold. To reduce the false alarm frequency caused by the pseudo-anomaly data, it needs to evaluate the reliability of the collected data. The main line of the Middle Route of the SNWTP is divided into several sections according to the geographical scope; the reliability of the data is judged by analyzing the relationship between similar and heterogeneous sensors in each section.
(1) For the same type of sensors in the same section, the data of the physical nodes in the sensor network are evaluated by the confidence level of the interval estimation based on the normal historical data. To analyze the linear/nonlinear correlation between the same types of sensors and set up the correlation model, when a sensor data is abnormal, it can be predicted by other similar sensors which have strong correlation with current sensor, which determines whether the current abnormal data is caused by a broken sensor or other reasons. The above data processing improves the reliability of the data with automatic detection.

(2) For the different types of sensors in the same section, when a sensor data is abnormal, strongly related similar or heterogeneous sensors can predict whether the data is correct by analyzing the correlation and trend of the data from many types of sensors and establishing the linear or nonlinear models among heterogeneous sensors, which improves the reliability of the warning data.

\section{Conclusions}

Due to the limitations of the bandwidth of network and coverage area of wireless network, the cloud computing framework fails to meet the requirements of real-time systems with high reliability. Fog computing transfers the computational tasks as much as possible to the fog servers deployed onsite, which not only reduces the overall time latency, but also provides computing services in an environment without an Internet connection. This work built the cloud/fog computing architecture for safety monitoring in water conservancy engineering, which reduced the limitation of high latency and high reliability constraints based on the cloud computing architecture. The IoT big data in water conservancy engineering with dense geographical distribution were applied based on multilevel requirements and integrated to a resource integration platform for deployment. It not only better meets the requirements of water conservancy project safety application, but also is more convenient for the development and deployment.

\section{Conflicts of Interest}

The authors declare that they have no conflicts of interest.

\section{Acknowledgments}

This work is supported by National Major Project "Research and Development of Sensor Network Technology Oriented to the Safety of the South-North Water Transfer Project (SNWTP)" (no. 2014ZX03005001).

\section{References}

[1] J. Gubbi, R. Buyya, S. Marusic, and M. Palaniswami, "Internet of Things (IoT): a vision, architectural elements, and future directions," Future Generation Computer Systems, vol. 29, no. 7, pp. 1645-1660, 2013.

[2] K. Hong, D. Lillethun, U. Ramachandran, B. Ottenwälder, and B. Koldehofe, "Mobile fog: a programming model for large-scale applications on the internet of things," in Proceedings of the 2nd 
ACM SIGCOMM Workshop on Mobile Cloud Computing (MCC '13), pp. 15-20, Hong Kong, China, August 2013.

[3] A. Al-Fuqaha, M. Guizani, M. Mohammadi, M. Aledhari, and M. Ayyash, "Internet of things: a survey on enabling technologies, protocols, and applications," IEEE Communications Surveys \& Tutorials, vol. 17, no. 4, pp. 2347-2376, 2015.

[4] M. Fazio, N. Bessis, and M. Villari, "Advances in serviceoriented and cloud computing," in Preface of CLIoT, vol. 58, pp. 73-75, Springer International Publishing, Berlin, Germany, 2015.

[5] A. Botta, W. De Donato, V. Persico, and A. Pescape, "On the integration of cloud computing and internet of things," in Proceedings of the 2nd International Conference on Future Internet of Things and Cloud (FiCloud '14), pp. 23-30, Barcelona, Spain, August 2014.

[6] T. Li and J. Han, Cloud Computing Architecture Technologies \& Practice, Qinghua University Press, Beijing, China, 2013.

[7] A. Sun, "Enabling collaborative decision-making in watershed management using cloud-computing services," Environmental Modeling and Software, vol. 41, pp. 93-97, 2013.

[8] C. M. Burger, S. Kollet, J. Schumacher et al., "Introduction of a web service for cloud computing with the integrated hydrologic simulation platform ParFlow," Computers \& Geosciences, vol. 48, pp. 334-336, 2012.

[9] I. Ari and N. Muhtaroglu, "Design and implementation of a cloud computing service for finite element analysis," Advances in Engineering Software, vol. 60-61, pp. 122-135, 2013.

[10] L.-X. Zhang and R.-M. Wang, "Research on the technology framework of water resources management information platfrom base on cloud computing," Journal of the Hebei Academy of Sciences, vol. 33, no. 2, pp. 12-16, 2016.

[11] R. J. Yang and Y. J. Yang, "Infrastructure design of water resources management system based on a private cloud computing," Journal of Guizhou University (Natural Sciences), vol. 30, no. 3, pp. 109-112, 2013.

[12] L. Zhou, W. Liu, and Y. Bai, “Technological study on cloud computing system and its general design for water scientific research," Journal of Yangtze River Scientific Research Institute, vol. 32, no. 11, pp. 119-124, 2015.

[13] Wikipedia, Fog computing [EB/OL], 2016, https://en.Wikipedia .org/wild/Fogcomputing.

[14] W. Steiner and S. Poledna, "Fog computing as enabler for the Industrial Internet of Things," Elektrotechnik und Informationstechnik, vol. 133, no. 7, pp. 310-314, 2016.

[15] W. Fang, "A paradigm shift to fog computing from cloud computing and edge computing," Journal of Nanjing University of Information Science and Technology: Natural Science Edition, vol. 8, no. 5, pp. 404-414, 2016.

[16] F. Bonomi, R. Milito, J. Zhu, and S. Addepalli, "Fog computing and its role in the internet of things," in Proceedings of the 1st edition of the ACM Mobile Cloud Computing Workshop on Mobile cloud Computing (MCC '12), pp. 13-15, Helsinki, Finland, August 2012.

[17] S. Tomovic, K. Yoshigoe, I. Maljevic, and I. Radusinovic, "Software-defined fog network architecture for IoT," Wireless Personal Communications, vol. 92, no. 1, pp. 181-196, 2017.

[18] M. Chiang and T. Zhang, "Fog and IoT: an overview of research opportunities," IEEE Internet of Things Journal, vol. 3, no. 6, pp. 854-864, 2016.

[19] T. V. N. Rao, M. A. Khan, M. Maschendra et al., "A paradigm shift from cloud to fog computing," IJCSET, vol. 5, no. 11, pp. 385-389, 2015.
[20] N. Peter, "Fog computing and its real time applications," International Journal of Emerging Technology and Advanced Engineering, vol. 5, no. 6, pp. 266-269, 2015. 


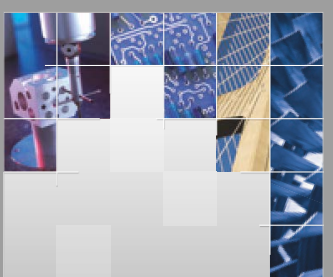

\section{Enfincering}
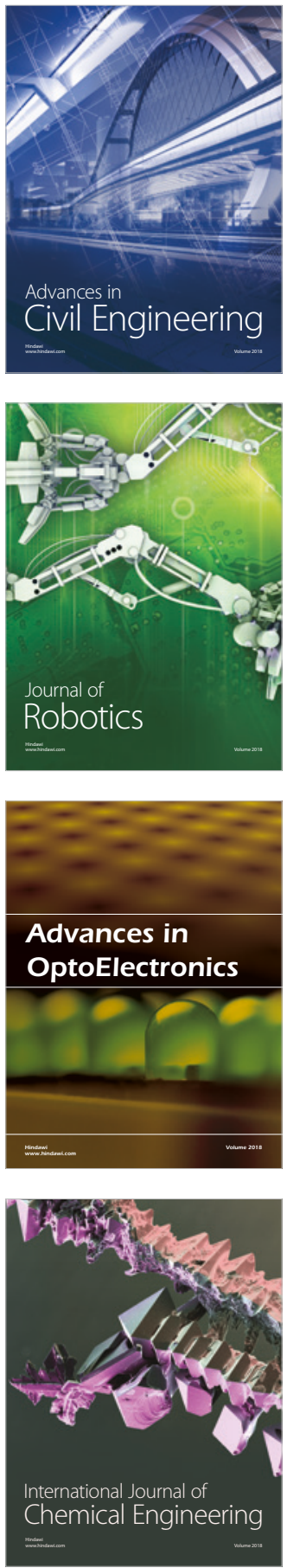

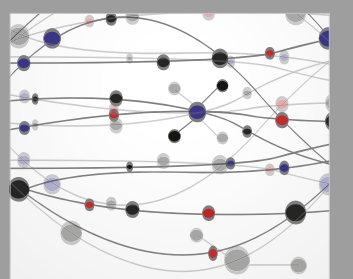

\section{Rotating \\ Machinery}

The Scientific World Journal

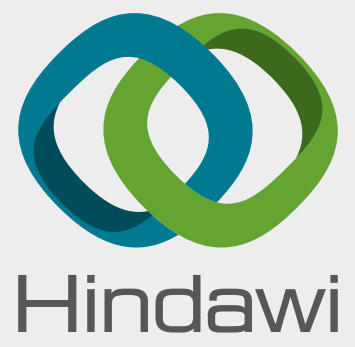

Submit your manuscripts at

www.hindawi.com
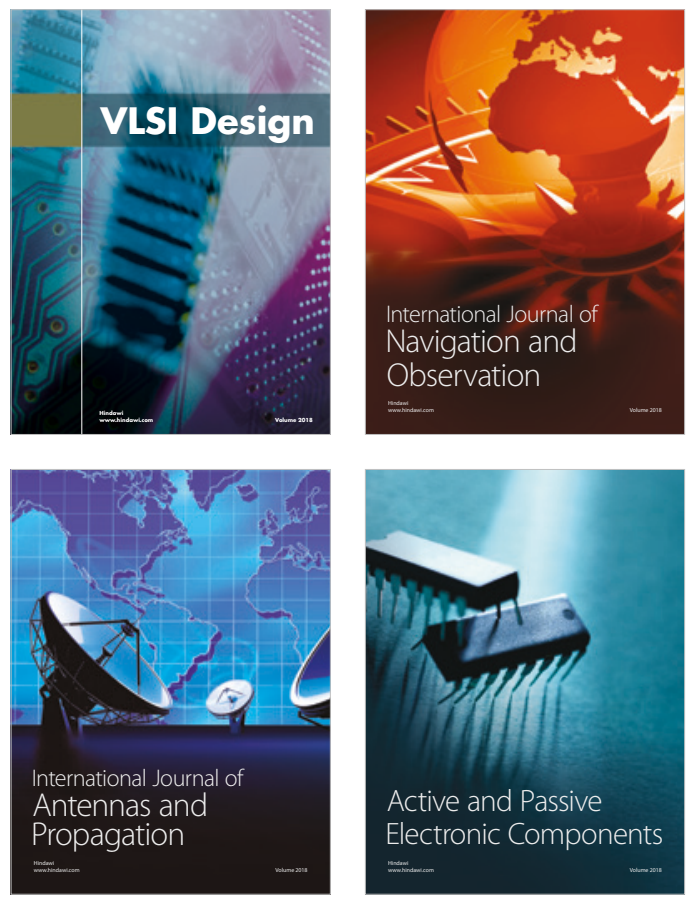
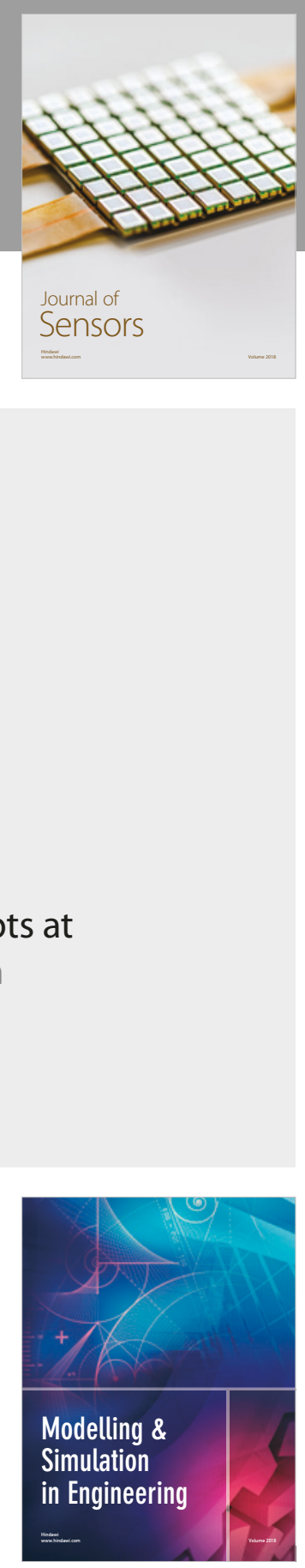

\section{Advances \\ Multimedia}
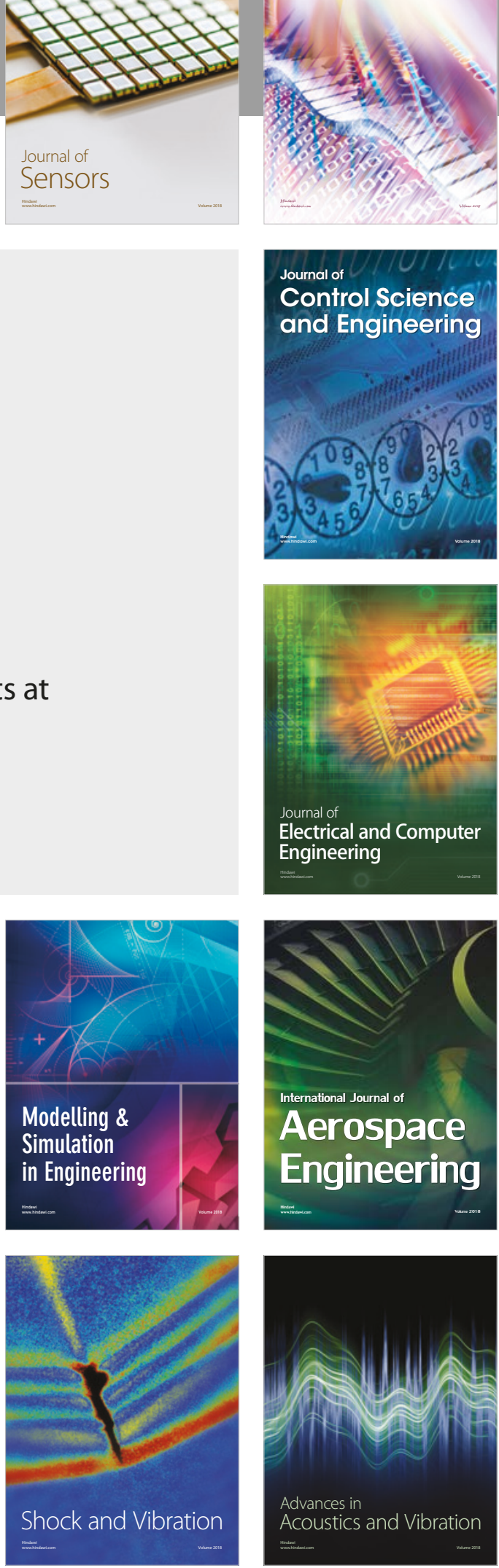